\title{
De Fazio, Federico. Teoría principialista de los derechos sociales. Madrid:
}

\section{Marcial Pons, 2019.}

\author{
Juan-Ramón Fallada-García-Valle \\ Universitat Rovira i Virgili
}

Fecha de recepción 26/11/2020 I De publicación: 28/12/2020

El libro de Federico de Fazio es, sin duda, de lectura recomendable. Goza de no pocas virtudes: coherencia discursiva, capacidad sintética, claridad y precisión expositiva, todo ello sin que vaya en detrimento de la fuerza y calado de los argumentos desplegados para sustentar la tesis que se propone defender. Junto a todas esas virtudes, seguramente la más destacada resida en que aporta un enfoque poco transitado y sugerente en lo que toca al estudio de los derechos sociales. Tal novedad no radica en la metodología empleada, la analítica, sino más bien en el empleo que hace de esta metodología para abordar el examen de las normas constitucionales de derecho social y extraer del mismo una fundamentación de su obligatoriedad jurídica (lo cual requiere previamente fundamentar su validez). De esta forma, se aparta del enfoque más recurrente que entiende los derechos sociales como derechos materiales, en contraposición al carácter formal de los derechos civiles y políticos.

Desde esas premisas analíticas, el punto de partida no podía ser otro que el de la constatación fáctica de la positivación de los derechos sociales en los textos constitucionales de no pocos Estados en la actualidad. La pregunta fundamental que hilvana los diversos capítulos es la de qué obligaciones jurídicas generan esos preceptos constitucionales que establecen derechos sociales. La secuencia argumental es impecable. La primera parte la dedica a las dos condiciones formales que fundamentarían la obligatoriedad de las normas de derecho social, a saber: 1) su validez, tarea a la que dedica el primer capítulo, y 2) el que una o varias cláusulas constitucionales ordene(n) realizar un derecho social en un grado exacto de cumplimiento, cuestión que afronta en el segundo capítulo. La segunda parte la dedica a los dos procedimientos argumentales que permitirían fundamentar la validez de una regla de derecho social en la medida en que ésta formaría parte del significado de una o varias cláusulas constitucionales. El capítulo tercero trata sobre aquellos argumentos en que no se requiere explicitar una relación de precedencia entre principios. El último capítulo trata sobre el tipo de argumentación al cual cabe recurrir 
cuando sí se requiere explicitar una relación de precedencia condicionada entre principios, centrando su interés concretamente en el de proporcionalidad por omisión.

En el primer capítulo aborda la cuestión de la validez de las normas constitucionales que establecen derechos sociales, en tanto que primer requisito para fundamentar su obligatoriedad. Para ello, irá tomando posición respecto a las cuestiones de qué es una norma, cuándo una norma es válida, qué normas de derecho social son constitucionales y qué son los derechos sociales.

Una vez ha aclarado que parte de una concepción semántica de las normas, define "norma" como el significado de una formulación normativa. De ahí deriva que la validez de una norma (esto es, de un mandato) dependa de su identificación con, al menos, una formulación normativa, ya sea explícita o implícita (esto es, cuando no se utilizan literalmente expresiones deónticas). Puntualiza que esa definición de validez vendría acotada por el propósito del trabajo, el cual, como se ha comentado, se dirige a fundamentar la obligatoriedad de los derechos sociales. A su vez, una norma de derecho social contaría con rango constitucional cuando puede ser fundamentada como parte del significado de, al menos, una cláusula o formulación constitucional. Evidentemente, no toda norma constitucional sería una norma de derecho social. Una norma constitucional sería una norma de derecho social sólo cuando su contenido ordena realizar un derecho social. De todo su razonamiento se desprende la conclusión esperada: las normas constitucionales de derecho social son válidas porque es posible identificar, al menos, una formulación normativa de rango constitucional.

Finalmente, en el primer capítulo también aclara qué entiende por “derecho social”. La definición viene condicionada por su voluntad de focalizarse en la problemática fundamental que suscitan los derechos sociales. Efectivamente, si bien los derechos sociales no agotarían su contenido en su dimensión prestacional fáctica, tal y como apunta de Fazio el meollo de la problemática jurídica de los derechos sociales reside en la obligatoriedad de esos contenidos. Sobre esas premisas, define "derecho social" como el "derecho subjetivo a una acción positiva fáctica". En otras palabras, por "derecho social" hace referencia a aquellos enunciados que, estructuralmente, establecen una relación triádica entre una persona física titular, un sujeto destinatario (que puede ser tanto el Estado, como una persona física o jurídica de derecho privado), y un derecho a que este último haga algo, en concreto, la entrega de un bien material o la prestación de un servicio. A diferencia de los derechos de no-intervención, los cuales responden siempre a estructuras conjuntivas (es decir, que solamente existe una medida idónea para lograr su cumplimiento), los derechos a una acción positiva fáctica pueden tener tanto una estructura conjuntiva 
como disyuntiva (esto es, cuando existe más de una única medida idónea para lograr su cumplimiento). Respecto a los derechos sociales con estructura disyuntiva, plantea la posible crítica de que, en realidad, los derechos sociales no generarían obligaciones, pues al no quedar especificada ninguna obligación en concreto del sujeto destinatario, éste no estaría obligado a realizar ninguna de las acciones alternativas presentadas por la norma. Frente a esta objeción, el autor replica que la posibilidad de que el sujeto destinatario pueda dar cumplimiento al derecho social de diversas maneras no significa que no tenga la obligación de dar cumplimiento, sino sólo que dispone de un cierto margen de discrecionalidad en el modo de darlo.

En el segundo capítulo entra a examinar la segunda condición necesaria para poder fundamentar en una decisión jurídica la exigibilidad de un derecho social constitucional: la necesidad de que una o varias cláusulas constitucionales ordene(n) realizar un derecho social en un grado exacto de cumplimiento.

Con ese propósito, comienza justificando la distinción entre reglas de derecho social y principios de derecho social. Las primeras serían todas aquellas normas constitucionales que ordenan realizar un derecho social en un grado exacto de cumplimiento. Las segundas serían todas aquellas normas constitucionales que ordenan optimizar un derecho social. En otras palabras, las reglas de derecho social consistirían en "mandatos de cumplimiento exacto", mientras que los principios de derecho social consistirían en "mandatos de optimización" al modo como los entiende Alexy (esto es, como la obligación de realizar el principio en cuestión en el mayor grado posible de acuerdo con las circunstancias fácticas y jurídicas existentes). La distinción entre reglas y principios residiría en que las reglas carecen de la dimensión del peso, mientras que los principios sí la poseerían. Esto implica que, en caso de contradicción entre reglas, ésta sólo puede ser solucionada o bien introduciendo una cláusula de excepción, o bien declarando la invalidez de, por lo menos, una de ellas. En cualquiera de esos casos, una de las dos reglas deja de operar como razón válida a tenerse en cuenta en la toma de decisiones (la contradicción sí se resuelve en la "dimensión de la validez”). En cambio, la prevalencia de un principio respecto a otro en un caso concreto no invalida al segundo. Así pues, los conflictos entre principios no se resuelven en la dimensión de la validez; bien al contrario, su validez persiste para la resolución de posibles casos futuros similares, o para futuros conflictos entre esos mismos principios que, ante circunstancias nuevas, puede llevar a la prevalencia del segundo principio sobre el primero. 
Así pues, considera que reglas y principios son cualitativamente distintos en función de su dimensión. Con todo, descarta que las reglas se distingan de los principios en que los primeros se caractericen por su aplicación a la manera de 'o todo o nada' y los segundos no. Para ello, por un lado, aduce que no es posible enumerar de antemano todos los supuestos de hecho en que una regla es de aplicación: la textura abierta del lenguaje y la asunción misma de una composición dual reglas-principios de los ordenamientos jurídicos lo impedirían. Por otro lado, y siguiendo nuevamente a Alexy, considera que, del conflicto entre principios en conexión con un caso concreto, se derivan reglas en las cuales el supuesto de hecho estaría conformado por las circunstancias fácticas que justifican una determinada relación de precedencia entre aquellos, configurando la consecuencia jurídica el principio que prevalece. En breve, los principios se concretarían en reglas mediante la resolución de casos judiciales, pero sin perder por ello su dimensión del peso. Dos apuntes más. Primero: los conflictos entre principios se resolverían mediante juicios de ponderación o proporcionalidad. Esas reglas derivadas de principios constitucionales de derecho social mediante juicios de proporcionalidad consistirían en normas indirectamente estatuidas. De esta forma, en la medida en que, mediante un juicio de ponderación, los principios se concretan en reglas indirectamente estatuidas, y que éstas, en tanto que reglas, consisten en mandatos de cumplimiento exacto, también los principios de derecho social cumplirían con la segunda condición exigida para su obligatoriedad.

Como se ha comentado, los capítulos tercero y cuarto los dedica a fundamentar la validez de las normas constitucionales de derecho social desde el plano de la argumentación jurídica. En las propias palabras del autor, desde este plano "una regla de derecho social sería válida cuando a su favor pueden darse argumentos que, en su debido contexto, deberían ser aceptados por cualquier participante de un discurso jurídico". De esta forma, redirige la cuestión a la indagación de los argumentos que, efectivamente, pueden respaldar suficientemente la validez de las reglas de derecho social para esos participantes.

El capítulo tercero se centra en los argumentos interpretativos, en el uso de precedentes y en el recurso a la analogía. Todos ellos tendrían en común que la argumentación desplegada no establece relaciones de precedencia entre principios respecto a un caso concreto. Este tipo de argumentos servirían para validar las reglas de derecho social, directa o indirectamente estatuidas.

Los argumentos interpretativos hallarían su fundamentación en una razón autoritativa, esto es, en que toda decisión jurídica debe estar justificada de acuerdo con el marco establecido por el derecho 
positivo sin acudir a cuestiones sustantivas de tipo moral y político. En contra de las objeciones que plantean quienes consideran que la fundamentación de reglas de derecho social siempre requiere de la fundamentación de las premisas morales y políticas que el juez está presuponiendo, de Fazio sostiene que, según el contexto de razonamiento jurídico, una sentencia puede estar perfectamente razonada sin otra ayuda que el empleo de argumentos autoritativos. Cuando ese fuera el caso, se establecería un argumento fuerte a favor de la validez de esa regla de derecho social, cayendo la carga de argumentar alguna posible excepción en base a algún principio del ordenamiento jurídico en conexión con un caso concreto. El principio de seguridad jurídica fundamentaría la prevalencia de la razón autoritativa y la carga de argumentar las excepciones a las reglas de derecho positivo.

El uso de precedentes validaría reglas de derecho social porque los precedentes consistirían en reglas que se pueden extraer de sentencias judiciales anteriores que justifican la regla derecho social empleada como premisa mayor en la argumentación del caso sometido a juicio. Si bien la fuerza vinculante de un precedente que valida una regla de derecho social variaría en función del sistema jurídico en cuestión, el órgano que lo emita y el nivel de racionalidad de sus fundamentos, habría una presunción prima facie a favor de su validez; en consecuencia, la carga de la argumentación correría de parte de quien se aparta de tal presunción. Por su parte, el recurso a la analogía consistiría en la regla que se puede extraer de una sentencia anterior respecto a un caso similar al caso sometido a juicio; en este punto, el recurso a la analogía se aproximaría al supuesto del uso de precedentes. En este punto precisa que, para considerar que dos casos son similares, tiene que haber un idéntico problema normativo y también una idéntica constelación de principios aplicables (que el conflicto entre principios sea idéntico).

El último capítulo es seguramente el más original y el que suscita mayor interés. En éste se propone fundamentar las obligaciones de prestación que contendrían los principios constitucionales de derecho social en base al procedimiento argumental del juicio de proporcionalidad.

En la medida en que los principios se concretan en reglas indirectamente estatuidas a través de los juicios de proporcionalidad, también es posible plantearse de qué modo este procedimiento argumental validaría esas reglas que estatuye indirectamente y, por lo tanto, también el principio de derecho social del cual se originaría. Concretamente, de Fazio se centra en el tipo de obligaciones que contendrían los principios de derecho social que más polémica suscitan, a saber, las obligaciones de prestación. Y es que, efectivamente, existe una fuerte controversia acerca de si realmente los principios de derecho social generan, en un sentido estricto, obligaciones de ese tipo. Ello le lleva a focalizar su 
análisis en los juicios de proporcionalidad por omisión, pues este tipo de juicios sería el adecuado para examinar una posible vulneración de ese tipo de obligaciones. El examen de proporcionalidad por omisión resultaría pertinente (y admisible) cuando se puede demostrar que el derecho social ha sido realizado en un grado menor al grado de su cumplimiento total o ideal como consecuencia de la ausencia de una o varias acciones positivas fácticas por parte de quien es el destinatario de la obligación establecida en el principio de derecho social.

El juicio de proporcionalidad por omisión gozaría de idéntica estructura que el juicio de proporcionalidad por exceso, es decir, que también estaría compuesto por los subexámenes de idoneidad, necesidad y proporcionalidad en sentido estricto. En buena medida, lo que sigue es una exposición del juicio de proporcionalidad, pero con la interesante novedad de su aplicación a aquellos supuestos en que se examina la omisión de una acción positiva fáctica de obligado cumplimiento prima facie con base en un principio de derecho social.

El subexamen de idoneidad consistiría en verificar que la omisión de una o varias acciones fácticas positivas limitativas de un principio de derecho social persigue un fin legítimo y que esa medida omisiva sea un medio adecuado para fomentar ese fin. Un fin sería ilegítimo cuando su promoción se encuentra definitivamente prohibida por una regla constitucional. Por otro lado, cuando una omisión de una o varias acciones positivas fácticas no contribuye en ningún grado y sentido al fomento de un fin legítimo, debe considerarse técnicamente inapropiada y, por tanto, desproporcionada. Si la medida omisiva sometida a juicio supera ese primer subexamen al considerarse idónea, se pasaría al siguiente subexamen.

El subexamen de necesidad consistiría en verificar que no existe alguna medida alternativa a la omisión de una o varias acciones positivas fácticas limitativas de un principio de derecho social que permita promover el fin legítimo por lo menos en el mismo grado y que resulta menos lesiva con respecto al principio de derecho social. Si tal medida alternativa existiera, entonces la omisión resultaría innecesaria y, por tanto, desproporcionada. Si tal medida no existiera, entonces la omisión sometida a juicio superaría también el subexamen de necesidad, procediéndose al subexamen de proporcionalidad en sentido estricto.

Finalmente, el subexamen de proporcionalidad en sentido estricto consistiría en verificar que toda limitación a un principio de derecho social como consecuencia de la omisión de una o varias acciones 
positivas fácticas genera más beneficios relativos en el fin que legitima esas medidas que los perjuicios que causa dicha limitación. Aquí el examen se traslada del plano de los medios y la facticidad al plano de la comparación del peso relativo en abstracto de los fines constitucionalmente legítimos en conflicto en conexión con un tipo de casos. La ley de ponderación la sintetiza en los términos siguientes: "cuanto mayor sea el valor compuesto por el peso relativo abstracto y el grado de limitación de un principio, tanto mayor debe ser el valor compuesto por el peso relativo abstracto y el grado de realización del otro". Si los perjuicios causados por la limitación a un principio de derecho social fueran mayores que los beneficios que genera para el fin legítimo perseguido por la omisión de la acción positiva fáctica sometida a evaluación, entonces tal omisión habría que considerarla desproporcionada. $\mathrm{Si}$, en cambio, los beneficios fueran mayores que los perjuicios, tal omisión habría que considerarla proporcionada y, por tanto, justificada.

Aunque por razones obvias no se puede aquí entrar en el detalle de su argumentación, por su particular relevancia creo que sí merece la pena exponer el modo cómo justifica el principio de no regresividad de los derechos sociales. A la hora de justificar la asignación de un peso relativo abstracto alto a un principio de derecho social, señala tres criterios implícitos: 1) la importancia de los intereses que dan fundamento a la validez del principio; 2) su asignación de peso relativo en abstracto 'alto' en decisiones anteriores; y 3) la conexión relevante del principio con otros principios. Los dos primeros criterios serían casos especiales del tercero. En cualquier caso, cabría asignar un peso relativo abstracto alto a un principio de derecho social cuando la omisión de una o varias acciones positivas fácticas vulnera dicho principio de no regresividad. Dicha conexión generaría una presunción prima facie en favor del principio de derecho social frente al fin legítimo que pretendidamente justificaría la medida lesiva.

De manera genérica, tanto la argumentación en base a los precedentes, como la argumentación analógica a partir de criterios considerados ya anteriormente relevantes, como la conexión de un principio de derecho social con otro principio que goza de un peso relativo abstracto alto dentro del juicio de proporcionalidad, todos ellos generan una presunción prima facie en su favor. En los dos primeros tipos de argumentación ello se traduce en que la carga de fundamentar, bien una solución contraria al precedente, bien la no consideración de un criterio considerado anteriormente relevante para fijar la analogía. En lo que se refiere a los juicios de proporcionalidad, la presunción prima facie en favor de un principio de derecho social se traduce en que la comparación del peso relativo entre fines constitucionalmente legítimos no sería lineal, sino progresivo en favor de esos principios que gozan de 
dicha presunción. De esta forma, introduce una corrección formal en favor del derecho positivo y la seguridad jurídica.

La argumentación que despliega a lo largo del trabajo para justificar la equiparación entre derechos sociales con los derechos civiles y políticos y la superación de la distinción derechos materiales frente a derechos formales resulta sólida con el propósito último de fundamentar la obligatoriedad de los derechos sociales reconocidos en las constituciones contemporáneas. En este punto, su recurso a una metodología analítica y formalista para lograr cumplir con ese propósito lo cumple perfectamente. Por otro lado, en mi opinión, el trabajo peca de las dificultades y las limitaciones típicas asociadas a esa metodología. A este respecto, destacaría dos puntos.

En primer lugar, más allá de la aparente neutralidad valorativa del método analítico, lo cierto es que esa opción metodológica parte de presupuestos valorativos que determinan los resultados alcanzados. A mi juicio, esto se pone particularmente de manifiesto en la comentada equiparación entre los derechos sociales respecto a los derechos civiles y políticos en tanto que derechos formales. Al no fundamentar la obligatoriedad de los mandatos de los derechos sociales en razones sustantivas que los relacionen abiertamente con la dignidad humana u otros similares, de ahí se deriva la inexistencia de unos mínimos sustantivos a garantizar, lo cual abre la puerta a su posible vaciamiento no sólo en situaciones consideradas extremas (catástrofes naturales, guerras, etc.), sino también en aquellos contextos con legisladores o aplicadores del derecho ideológicamente opuestos a su reconocimiento. Y es que la fuerza argumentativa atribuida a los principios constitucionales de derecho social en los procesos legislativos y jurisdiccionales depende ulteriormente del marco ideológico-valorativo desde el cual son evaluados. En definitiva, si se deslinda la fundamentación de los derechos sociales de todo valor sustantivo, se abre la posibilidad de su vaciamiento, consecuencia que, paradójicamente, parece la opuesta a la que el autor pretende sustentar. Finalmente, esta dificultad no se sortea apelando a una suerte de participante "racional" de un discurso jurídico que debería aceptar ciertos argumentos en favor del no vaciamiento de los principios constitucionales de derecho social sin aclarar de qué modo esa apelación no se sustenta sobre una manera de entender la racionalidad de tipo sustantivo y sí sobre una manera de entender la racionalidad puramente lógico-formal (o procedimental).

En segundo lugar y conectado con el punto anterior, aunque el trabajo pretende describir los ordenamientos jurídicos contemporáneos, su análisis se mueve inevitablemente en un grado de abstracción tal que resulta inviable captar la diversidad de realidades jurídicas. Efectivamente, al 
comienzo de su libro afirma tomar como punto de partida fáctico la positivación de los derechos sociales en las constituciones actuales. Pero, a mi entender, caben serias dudas respecto a la pertinencia o, cuando menos, respecto al alcance de la tesis defendida por de Fazio sobre la obligatoriedad de los principios constitucionales de derecho social en el caso de constituciones que incluyen cláusulas como la estipulada, por ejemplo, en el artículo 53.3 de la constitución española (el cual reza: "el reconocimiento, el respeto y la protección de los principios reconocidos en el Capítulo Tercero -esto es, la mayoría de principios constitucionales de derecho social [el inciso es mío]- informarán la legislación positiva, la práctica judicial y la actuación de los poderes públicos. Sólo podrán ser alegados ante la Jurisdicción ordinaria de acuerdo con lo que dispongan las leyes que los desarrollen”). En este tipo de cláusulas, explícitamente se exige el desarrollo legislativo de los principios constitucionales de derecho social de cara a poder generar obligaciones jurídicas susceptibles de ser invocadas ante los tribunales. En estas constituciones, el alcance de la tesis parece quedar limitado a aquellos derechos sociales que puedan ser directamente invocados, ya sea porque gozan de por sí de mayores garantías jurisdiccionales (como sería el caso, en la constitución española, del derecho a la educación), o indirectamente porque se han establecido conexiones con otros derechos constitucionales que sí gozan de esas mayores garantías. Si no se da ninguno de los dos supuestos anteriores, entonces el alcance del análisis puede verse supeditado a que efectivamente haya habido tal desarrollo legislativo, así como lastrado por el rango infraconstitucional de las obligaciones generadas por los principios de derecho social. 